\title{
Note on Transliteration and Spelling of Non-English Words
}

\begin{abstract}
The non-English vocabulary in this study draws on Tamil, Sanskrit, Urdu, and Anglo-Indian sources. Many terms used in written documents vary in their original transcription. In cases where a word occurs in a Tamil document or was spoken by a Tamil informant, I severely modify the transliteration system of the Madras University Lexicon, stripping terms of diacritical marks that would be useful only for Tamil scholars. In addition, plural forms of these terms, when used in the text rather than mentioned in a quotation from Tamil, are formed by adding the English morpheme $s$ (e.g., panams rather than panankal). In cases where a word occurs in governmental or other documents from the colonial period, I adopt its most frequent Anglo-Indian spelling. In cases where a word occurs in both contexts, I generally follow the Anglo-Indian conventions, especially with regard to proper names. For variant transcriptions of the terms and for diacritical marks providing a precise indication of non-English orthography or phonology, see the glossary.
\end{abstract}


